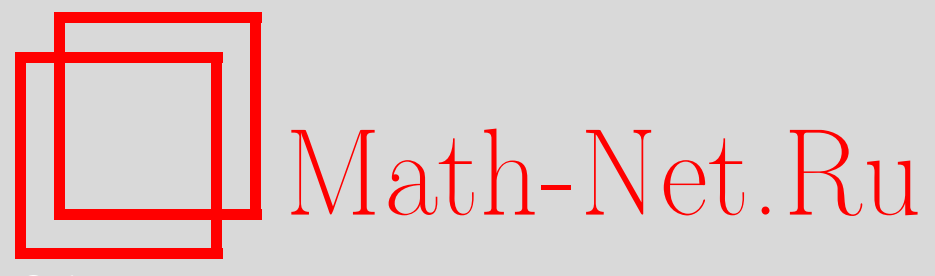

А. А. Домнич, Е. С. Барановский, М. А. Артёмов, О математической модели неизотермического ползущего течения жидкости через заданную область, Вестн. Сам. гос. техн. ун-та. Сер. Физ.-мат. науки, 2019, номер 3, 417-429

DOI: https://doi.org/10.14498/vsgtu1713

Использование Общероссийского математического портала MathNet.Ru подразумевает, что вы прочитали и согласны с пользовательским соглашением

http://www.mathnet.ru/rus/agreement

Параметры загрузки:

IP : 54.89 .56 .158

26 апреля 2023 г., $14: 26: 25$

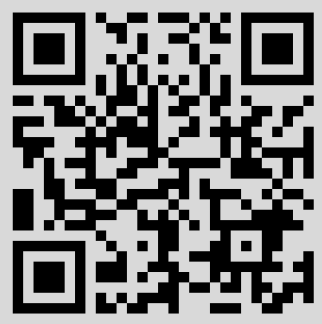


УДК 517.958:531.32

\title{
О математической модели неизотермического ползущего течения жидкости через заданную область
}

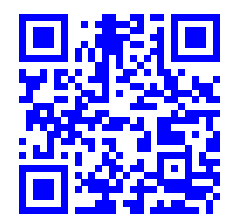

\author{
А. А. Домнич, Е. С. Барановский ${ }^{2}$, М. А. Артемов ${ }^{2}$ \\ 1 Военный учебно-научный центр Военно-воздушных сил \\ «Военно-воздушная академия им. профессора Н. Е. Жуковского и Ю. А. Гагарина» \\ Россия, 394064, Воронеж, ул. Старых большевиков, 54 а. \\ 2 Воронежский государственный университет, \\ Россия, 394018, Воронеж, Университетская пл., 1.
}

\section{Аннотация}

Рассматривается математическая модель, описывающая стационарное ползущее течение неравномерно нагретой несжимаемой жидкости через ограниченную трехмерную область с локально-липшицевой границей. Выбранная модель представляет собой систему нелинейных дифференциальных уравнений в частных производных второго порядка со смешанными краевыми условиями: на участке протекания заданы давление, температура и касательная составляющая поля скоростей, а на твердых стенках сосуда используется условие прилипания и краевое условие типа Робена для температуры. Для данной краевой задачи вводится понятие слабого решения (пара «скорость-температура»), которое определяется как решение некоторой системы интегральных тождеств. Основной результат работы - теорема о существовании слабых решений в подпространстве декартова произведения двух соболевских пространств. Для доказательства этой теоремы дается операторная трактовка краевой задачи, выводятся априорные оценки решений и применяется теорема Лерэ-Шаудера о неподвижной точке вполне непрерывного отображения. Установлены энергетические равенства, которым удовлетворяют слабые решения.

Ключевые слова: задача протекания, неизотермическое течение, ползущее течение, смешанные краевые условия, слабые решения.

\section{Научная статья}

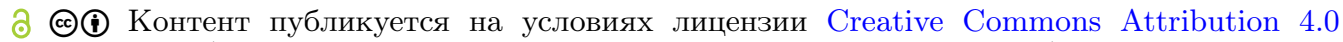
International (https://creativecommons.org/licenses/by/4.0/deed.ru)

\section{Образец для цитирования}

Домн ич А. А., Баранов ск ий Е. С., А р тем ов М. А. О математической модели неизотермического ползущего течения жидкости через заданную область // Вестн. Сам. гос. техн. ун-та. Сер. Физ.-мат. науки, 2019. Т. 23, № 3. С. 417-419. doi: 10.14498/vsgtu1713.

\section{Сведения об авторах}

Анастасия Александровна Домнич (D) https://orcid.org/0000-0001-5231-4907

преподаватель; каф. высшей математики; e-mail: andomnich@inbox.ru

Евгений Сергеевич Барановский (1) https://orcid.org/0000-0002-1514-4475

кандидат физико-математических наук; доцент; факультет прикладной математики, информатики и механики; e-mail: esbaranovskii@gmail.com

Михаил Анатольевич Артемов (1) https://orcid.org/0000-0001-8356-5418 доктор физико-математических наук, профессор; заведующий кафедрой; факультет прикладной математики, информатики и механики; e-mail: artemov_m_a@mail.ru 
Получение: 15 июня 2019 г. / Исправление: 14 июля 2019 г.

Принятие: 16 сентября 2019 г. / Публикация онлайн: 14 октября 2019 г.

Введение. Пусть $\Omega \subset \mathbb{R}^{3}$ - ограниченная область с локально-липшицевой границей $\partial \Omega$. Рассмотрим стационарную математическую модель, описывающую неизотермическое течение вязкой несжимаемой жидкости (в безынерциальном приближении) через область $\Omega$ при смешанных краевых условиях:

$$
\left\{\begin{array}{l}
-\mu \Delta \boldsymbol{u}+\nabla p=\boldsymbol{f}(\boldsymbol{x}, \theta) \text { в } \Omega, \\
\operatorname{div} \boldsymbol{u}=0 \text { в } \Omega, \\
(\boldsymbol{u} \cdot \nabla) \theta-\kappa \Delta \theta=\varphi(\boldsymbol{x}, \theta) \text { в } \Omega, \\
\boldsymbol{u}=\mathbf{0}, \quad \kappa \frac{\partial \theta}{\partial \mathbf{n}}=-\alpha \theta \text { на } \partial \Omega \backslash S, \\
\boldsymbol{u}_{\tau}=\mathbf{0}, \quad p=p_{0}+C, \quad \theta=0 \text { на } S,
\end{array}\right.
$$

где $\boldsymbol{u}=\boldsymbol{u}(\boldsymbol{x})$ - скорость течения в точке $\boldsymbol{x} \in \Omega ; \theta=\theta(\boldsymbol{x})$ - отклонение температуры от равновесного значения; $\boldsymbol{f}(\boldsymbol{x}, \theta)$ - внешние силы; $p=p(\boldsymbol{x})$ - давление; $\mu>0-$ коэффициент вязкости; $\kappa>0-$ коэффициент теплопроводности; $\alpha>0$ - коэффициент теплообмена на стенках сосуда $\Omega ; \varphi(\boldsymbol{x}, \theta)$ - мощность тепловых источников; $\mathbf{n}=\mathbf{n}(\boldsymbol{x})$ - единичная внешняя нормаль к $\partial \Omega$; $S$ - плоский участок границы $\partial \Omega$, где происходит протекание жидкости (или объединение нескольких таких участков); $p_{0}$ - функция, характеризующая давление на $S$. Нижний индекс $\tau$ используется для обозначения касательной составляющей вектора, т. е. $\boldsymbol{u}_{\tau}:=\boldsymbol{u}-(\boldsymbol{u} \cdot \mathbf{n}) \mathbf{n}$. Произвольная константа $C$ в пятой строке системы (А) отражает факт, что при задании давления важен только его перепад. Краевое условие Дирихле для функции $\theta$ на участке протекания $S$ выбрано однородным лишь для упрощения математических выкладок и может быть заменено соответствующим неоднородным условием.

Основная цель данной работы - обоснование разрешимости задачи (А) в слабой формулировке. Доказательство теоремы существования основано на получении априорных оценок слабых решений и применении теоремы ЛерэШаудера о неподвижной точке вполне непрерывного отображения.

Следует отметить, что в линейной постановке задача о протекании неравномерно нагретой вязкой жидкости сквозь заданную область рассмотрена С. Г. Крейном и Чан Тху Ха [1]. В статьях [2,3] исследуются стационарные модели, описывающие (в рамках приближения Буссинеска) движение высоковязкой жидкости внутри ограниченной области с непроницаемыми твердыми стенками. Вопросы оптимизации и управления течением теплопроводной жидкости рассматриваются в [4-8]. В недавних публикациях $[9,10]$ установлены условия однозначной разрешимости двумерных эволюционных уравнений Обербека-Буссинеска с коэффициентом вязкости, зависящим от температуры. Задача Коши для уравнений Эйлера-Буссинеска, моделирующих потоки идеальной $(\mu \equiv 0)$ неравномерно нагретой жидкости, рассматривается в [11]. Отметим также статьи [12,13], в которых обсуждаются точные решения для описания установившихся ползущих конвективных течений несжимаемой жидкости, а также работы $[14,15]$, посвященные анализу крупномасштабной 
слоистой стационарной конвекции вязкой жидкости под действием касательных напряжений на верхней границе.

Классические уравнения Стокса и Навье-Стокса с краевыми условиями для давления $p$ или напора жидкости $p+|\boldsymbol{u}|^{2} / 2$ изучались многими авторами (см., например, [16-19] и цитируемую там литературу).

Основные допущения. Будем предполагать, что:

(C1) множества $S$ и $\partial \Omega \backslash S$ имеют положительную двумерную меру Лебега;

(C2) функция $p_{0}: S \rightarrow \mathbb{R}$ принадлежит пространству Лебега $L^{2}(S)$;

(C3) функции $f_{i}(\cdot, y): \Omega \rightarrow \mathbb{R}, i=\overline{1,3}$, и $\varphi(\cdot, y): \Omega \rightarrow \mathbb{R}$ измеримы при любом $y \in \mathbb{R}$

(C4) функции $f_{i}(\boldsymbol{x}, \cdot): \mathbb{R} \rightarrow \mathbb{R}, i=\overline{1,3}$, и $\varphi(\boldsymbol{x}, \cdot): \mathbb{R} \rightarrow \mathbb{R}$ непрерывны при п. в. $\boldsymbol{x} \in \Omega$;

(C5) существуют функция $f_{0} \in L^{2}(\Omega)$ и константа $M>0$ такие, что

$$
|\boldsymbol{f}(\boldsymbol{x}, y)| \leqslant f_{0}(\boldsymbol{x})+M|y|^{2}
$$

для любого $y \in \mathbb{R}$ и п. в. $\boldsymbol{x} \in \Omega$;

(C6) существует функция $\varphi_{0} \in L^{2}(\Omega)$ такая, что

$$
|\varphi(\boldsymbol{x}, y)| \leqslant \varphi_{0}(\boldsymbol{x})
$$

для любого $y \in \mathbb{R}$ и п. в. $\boldsymbol{x} \in \Omega$.

Слабая формулировка задачи (А). Сначала введем необходимые обозначения и функциональные пространства. В данной заметке используются пространства Лебега $L^{r}(\Omega), L^{r}(\partial \Omega)$, где $r \geqslant 1$, и пространство Соболева $H^{1}(\Omega):=W_{2}^{1}(\Omega)$. Соответствующие классы вектор-функций будем обозначать теми же символами, но при этом будем использовать жирный шрифт, т. е. $\boldsymbol{L}^{r}(\Omega):=L^{r}(\Omega)^{3}, \boldsymbol{L}^{r}(\partial \Omega):=L^{r}(\partial \Omega)^{3}, \boldsymbol{H}^{1}(\Omega):=H^{1}(\Omega)^{3}$. Пусть

$$
\begin{aligned}
\boldsymbol{V}_{S}(\Omega) & :=\left\{\boldsymbol{v} \in \boldsymbol{H}^{1}(\Omega): \operatorname{div} \boldsymbol{v}=0,\left.\boldsymbol{v}\right|_{\partial \Omega \backslash S}=\mathbf{0},\left.\boldsymbol{v}_{\tau}\right|_{S}=\mathbf{0}\right\}, \\
Y_{S}(\Omega) & :=\left\{\eta \in H^{1}(\Omega):\left.\eta\right|_{S}=0\right\} .
\end{aligned}
$$

Напомним, что сужение функции $w \in H^{1}(\Omega)$ на $\partial \Omega$ определяется по формуле $\left.w\right|_{\partial \Omega}:=\gamma_{\partial \Omega} w$, где $\gamma_{\partial \Omega}: H^{1}(\Omega) \rightarrow L^{4}(\partial \Omega)$ - оператор следа (см., например, [20, $§ 2.4 .2])$.

В пространстве $\boldsymbol{V}_{S}(\Omega)$ введем скалярное произведение и норму по формулам

$$
(\boldsymbol{v}, \boldsymbol{w})_{\boldsymbol{V}_{S}(\Omega)}:=\int_{\Omega} \nabla \boldsymbol{v}: \nabla \boldsymbol{w} \boldsymbol{d} \boldsymbol{x}, \quad\|\boldsymbol{v}\|_{\boldsymbol{V}_{S}(\Omega)}:=(\boldsymbol{v}, \boldsymbol{v})_{\boldsymbol{V}_{S}(\Omega)}^{1 / 2},
$$

где символ «:» обозначает скалярное произведение соответствующих матриц. Используя неравенство Фридрихса (см., например, [20, §1.1.8, теорема 1.9]) и условие (C1), нетрудно убедиться в том, что данное определение корректно и норма $\|\cdot\|_{\boldsymbol{V}_{S}(\Omega)}$ эквивалентна норме, индуцированной из $\boldsymbol{H}^{1}(\Omega)$.

В пространстве $Y_{S}(\Omega)$ зададим скалярное произведение и норму по формулам:

$$
(\eta, \zeta)_{Y_{S}(\Omega)}:=\int_{\Omega} \nabla \eta \cdot \nabla \zeta \boldsymbol{d} \boldsymbol{x}, \quad\|\eta\|_{Y_{S}(\Omega)}:=(\eta, \eta)_{Y_{S}(\Omega)}^{1 / 2} .
$$


Из неравенства Фридрихса и условия (C1) следует, что это определение корректно и норма $\|\cdot\|_{Y_{S}(\Omega)}$ эквивалентна стандартной $H^{1}$-норме.

Теперь мы можем дать слабую формулировку краевой задачи (А).

ОПРедЕлЕниЕ. Назовем слабым решением краевой задачи (А) пару функций $(\boldsymbol{u}, \theta) \in \boldsymbol{V}_{S}(\Omega) \times Y_{S}(\Omega)$ такую, что

$$
\begin{gathered}
\mu \int_{\Omega} \nabla \boldsymbol{u}: \nabla \boldsymbol{v} \boldsymbol{d} \boldsymbol{x}+\int_{S} p_{0}(\boldsymbol{v} \cdot \mathbf{n}) d \sigma=\int_{\Omega} \boldsymbol{f}(\boldsymbol{x}, \theta) \cdot \boldsymbol{v} \boldsymbol{d} \boldsymbol{x} \\
-\sum_{i=1}^{3} \int_{\Omega} u_{i} \theta \frac{\partial \eta}{\partial x_{i}} \boldsymbol{d} \boldsymbol{x}+\kappa \int_{\Omega} \nabla \theta \cdot \nabla \eta \boldsymbol{d} \boldsymbol{x}+\alpha \int_{\partial \Omega \backslash S} \theta \eta d \sigma=\int_{\Omega} \varphi(\boldsymbol{x}, \theta) \eta \boldsymbol{d} \boldsymbol{x}
\end{gathered}
$$

для любых $\boldsymbol{v} \in \boldsymbol{V}_{S}(\Omega)$ и $\eta \in Y_{S}(\Omega)$.

ЗАмечАниЕ. Покажем, каким образом в определении слабых решений возникают тождества $(1)$ и $(2)$. Пусть тройка $(\boldsymbol{u}, \theta, p)$ - классическое решение задачи $(\mathrm{A})$. Умножим скалярно в $\boldsymbol{L}^{2}(\Omega)$ обе части первого равенства из $(\mathrm{A})$ на вектор-функцию $\boldsymbol{v} \in \boldsymbol{V}_{S}(\Omega)$ :

$$
-\underbrace{\int_{\Omega} \mu \Delta \boldsymbol{u} \cdot \boldsymbol{v} \boldsymbol{d} \boldsymbol{x}}_{I_{1}}+\underbrace{\int_{\Omega} \nabla p \cdot \boldsymbol{v} \boldsymbol{d} \boldsymbol{x}}_{I_{2}}=\int_{\Omega} \boldsymbol{f}(\boldsymbol{x}, \theta) \cdot \boldsymbol{v} \boldsymbol{d} \boldsymbol{x} .
$$

Интегрированием по частям получаем

$$
I_{1}=\mu \int_{\partial \Omega} \frac{\partial \boldsymbol{u}}{\partial \mathbf{n}} \cdot \boldsymbol{v} d \sigma-\mu \int_{\Omega} \nabla \boldsymbol{u}: \nabla \boldsymbol{v} \boldsymbol{d} \boldsymbol{x}
$$

откуда, принимая во внимание соотношения

$$
\boldsymbol{v}=(\boldsymbol{v} \cdot \mathbf{n}) \mathbf{n} \text { на } S, \quad \boldsymbol{v}=\mathbf{0} \text { на } \partial \Omega \backslash S,
$$

находим, что

$$
I_{1}=\mu \int_{S} \frac{\partial(\boldsymbol{u} \cdot \mathbf{n})}{\partial \mathbf{n}}(\boldsymbol{v} \cdot \mathbf{n}) d \sigma-\mu \int_{\Omega} \nabla \boldsymbol{u}: \nabla \boldsymbol{v} \boldsymbol{d} \boldsymbol{x} .
$$

Поскольку $\operatorname{div} \boldsymbol{u}=0$ в $\Omega$ и $\boldsymbol{u}_{\tau}=0$ на $S$, то

$$
\frac{\partial(\boldsymbol{u} \cdot \mathbf{n})}{\partial \mathbf{n}}=0 \quad \text { на } S
$$

Поэтому

$$
I_{1}=-\mu \int_{\Omega} \nabla \boldsymbol{u}: \nabla \boldsymbol{v} \boldsymbol{d} \boldsymbol{x} .
$$

Далее, используя интегрирование по частям и равенства

$$
\operatorname{div} \boldsymbol{v}=0 \text { в } \Omega, \quad \int_{S} \boldsymbol{v} \cdot \mathbf{n} d \sigma=0, \quad \boldsymbol{v}=\mathbf{0} \text { на } \partial \Omega \backslash S, \quad p=p_{0}+C \text { на } S,
$$


получаем

$$
I_{2}=\int_{\partial \Omega} p(\boldsymbol{v} \cdot \mathbf{n}) d \sigma-\int_{\Omega} p \operatorname{div} \boldsymbol{v} \boldsymbol{d} \boldsymbol{x}=\int_{S}\left(p_{0}+C\right)(\boldsymbol{v} \cdot \mathbf{n}) d \sigma=\int_{S} p_{0}(\boldsymbol{v} \cdot \mathbf{n}) d \sigma .
$$

Подставляя (4) и (5) в равенство (3), приходим к (1). Аналогично, умножим скалярно в $L^{2}(\Omega)$ второе уравнение $(\mathrm{A})$ на $\eta \in Y_{S}(\Omega)$. С помощью интегрирования по частям и соответствующих краевых условий получаем (2).

Теорема о разрешимости задачи (А). Основным результатом работы является следующая

Теорема. Пусть выполнены условия (C1)-(C6). Тогда:

- задача (A) имеет по крайней мере одно слабое решение;

- всякое слабое решение задачи (A) удовлетворяет следующим энергетическим равенствам:

$$
\begin{gathered}
\mu \int_{\Omega}|\nabla \boldsymbol{u}|^{2} \boldsymbol{d} \boldsymbol{x}+\int_{S} p_{0}(\boldsymbol{u} \cdot \mathbf{n}) d \sigma=\int_{\Omega} \boldsymbol{f}(\boldsymbol{x}, \theta) \cdot \boldsymbol{u} \boldsymbol{d} \boldsymbol{x}, \\
\kappa \int_{\Omega}|\nabla \theta|^{2} \boldsymbol{d} \boldsymbol{x}+\alpha \int_{\partial \Omega \backslash S}|\theta|^{2} d \sigma=\int_{\Omega} \varphi(\boldsymbol{x}, \theta) \theta \boldsymbol{d} \boldsymbol{x} .
\end{gathered}
$$

Доказательство. Для доказательства существования слабых решений воспользуемся теоремой Лерэ-Шаудера о неподвижной точке вполне непрерывного отображения (см., например, $[21$, гл. I, §3]). С этой целью рассмотрим следующую однопараметрическую вспомогательную задачу.

Требуется найти пару $(\boldsymbol{u}, \theta) \in \boldsymbol{V}_{S}(\Omega) \times Y_{S}(\Omega)$, удовлетворяющую равенствам:

$$
\begin{aligned}
\mu \int_{\Omega} \nabla \boldsymbol{u}: \nabla \boldsymbol{v} \boldsymbol{d} \boldsymbol{x}+\lambda \int_{S} p_{0}(\boldsymbol{v} \cdot \mathbf{n}) d \sigma=\lambda \int_{\Omega} \boldsymbol{f}(\boldsymbol{x}, \theta) \cdot \boldsymbol{v} \boldsymbol{d} \boldsymbol{x} \quad \forall \boldsymbol{v} \in \boldsymbol{V}_{S}(\Omega), \\
-\lambda \sum_{i=1}^{3} \int_{\Omega} u_{i} \theta \frac{\partial \eta}{\partial x_{i}} \boldsymbol{d} \boldsymbol{x}+\kappa \int_{\Omega} \nabla \theta \cdot \nabla \eta \boldsymbol{d} \boldsymbol{x}+\alpha \int_{\partial \Omega \backslash S} \theta \eta d \sigma=\lambda \int_{\Omega} \varphi(\boldsymbol{x}, \theta) \eta \boldsymbol{d} \boldsymbol{x} \\
\forall \eta \in Y_{S}(\Omega),
\end{aligned}
$$

где $\lambda$ - параметр, $\lambda \in[0,1]$.

Установим априорные оценки решений задачи (8), (9). Предположим, что пара $(\boldsymbol{u}, \theta) \in \boldsymbol{V}_{S}(\Omega) \times Y_{S}(\Omega)$ удовлетворяет (8), (9) при некотором $\lambda \in[0,1]$. Полагая в равенстве $(8) \boldsymbol{v}=\boldsymbol{u}$, получаем

$$
\mu \int_{\Omega}|\nabla \boldsymbol{u}|^{2} \boldsymbol{d} \boldsymbol{x}+\lambda \int_{S} p_{0}(\boldsymbol{u} \cdot \mathbf{n}) d \sigma=\lambda \int_{\Omega} \boldsymbol{f}(\boldsymbol{x}, \theta) \cdot \boldsymbol{u} \boldsymbol{d} \boldsymbol{x},
$$

откуда с помощью условия (C5) и неравенства Коши-Буняковского выводим, что

$$
\mu\|\boldsymbol{u}\|_{\boldsymbol{V}_{S}(\Omega)}^{2}=\mu \int_{\Omega}|\nabla \boldsymbol{u}|^{2} \boldsymbol{d} \boldsymbol{x}=-\lambda \int_{S} p_{0}(\boldsymbol{u} \cdot \mathbf{n}) d \sigma+\lambda \int_{\Omega} \boldsymbol{f}(\boldsymbol{x}, \theta) \cdot \boldsymbol{u} \boldsymbol{d} \boldsymbol{x} \leqslant
$$




$$
\begin{aligned}
& \leqslant \int_{S}\left|p_{0}\right||\boldsymbol{u}| d \sigma+\int_{\Omega}\left(f_{0}+M|\theta|^{2}\right)|\boldsymbol{u}| \boldsymbol{d} \boldsymbol{x} \leqslant \\
& \begin{aligned}
\leqslant\left(\int_{S}\left|p_{0}\right|^{2} d \sigma\right)^{1 / 2}\left(\int_{S}|\boldsymbol{u}|^{2} d \sigma\right)^{1 / 2} & +\left(\int_{\Omega}\left|f_{0}\right|^{2} \boldsymbol{d} \boldsymbol{x}\right)^{1 / 2}\left(\int_{\Omega}|\boldsymbol{u}|^{2} \boldsymbol{d} \boldsymbol{x}\right)^{1 / 2}+ \\
& +M\left(\int_{\Omega}|\theta|^{4} \boldsymbol{d} \boldsymbol{x}\right)^{1 / 2}\left(\int_{\Omega}|\boldsymbol{u}|^{2} \boldsymbol{d} \boldsymbol{x}\right)^{1 / 2}=
\end{aligned} \\
& =\left\|p_{0}\right\|_{L^{2}(S)}\|\boldsymbol{u}\|_{\boldsymbol{L}^{2}(S)}+\left\|f_{0}\right\|_{L^{2}(\Omega)}\|\boldsymbol{u}\|_{\boldsymbol{L}^{2}(\Omega)}+M\|\theta\|_{L^{4}(\Omega)}^{2}\|\boldsymbol{u}\|_{\boldsymbol{L}^{2}(\Omega)} .
\end{aligned}
$$

Заметим, что существуют постоянные $C_{i}(\Omega, S), i=\overline{1,4}$, такие, что

$$
\begin{array}{cl}
\|\boldsymbol{u}\|_{\boldsymbol{L}^{2}(S)} \leqslant C_{1}(\Omega, S)\|\boldsymbol{u}\|_{\boldsymbol{V}_{S}(\Omega)}, & \|\boldsymbol{u}\|_{\boldsymbol{L}^{2}(\Omega)} \leqslant C_{2}(\Omega, S)\|\boldsymbol{u}\|_{\boldsymbol{V}_{S}(\Omega)}, \\
\|\theta\|_{L^{4}(\Omega)} \leqslant C_{3}(\Omega, S)\|\theta\|_{Y_{S}(\Omega)}, & \|\theta\|_{L^{2}(\Omega)} \leqslant C_{4}(\Omega, S)\|\theta\|_{Y_{S}(\Omega)} .
\end{array}
$$

Поэтому (11) можно продолжить следующим образом:

$$
\begin{array}{r}
\mu\|\boldsymbol{u}\|_{\boldsymbol{V}_{S}(\Omega)}^{2} \leqslant C_{1}(\Omega, S)\left\|p_{0}\right\|_{L^{2}(S)}\|\boldsymbol{u}\|_{\boldsymbol{V}_{S}(\Omega)}+C_{2}(\Omega, S)\left\|f_{0}\right\|_{L^{2}(\Omega)}\|\boldsymbol{u}\|_{\boldsymbol{V}_{S}(\Omega)}+ \\
+M C_{2}(\Omega, S) C_{3}^{2}(\Omega, S)\|\theta\|_{Y_{S}(\Omega)}^{2}\|\boldsymbol{u}\|_{\boldsymbol{V}_{S}(\Omega)}
\end{array}
$$

откуда следует

$$
\begin{aligned}
\|\boldsymbol{u}\|_{\boldsymbol{V}_{S}(\Omega)} \leqslant \mu^{-1} C_{1}(\Omega, S)\left\|p_{0}\right\|_{L^{2}(S)}+ & \mu^{-1} C_{2}(\Omega, S)\left\|f_{0}\right\|_{L^{2}(\Omega)}+ \\
& +\mu^{-1} M C_{2}(\Omega, S) C_{3}^{2}(\Omega, S)\|\theta\|_{Y_{S}(\Omega)}^{2}
\end{aligned}
$$

Положим теперь в (9) $\eta=\theta$ :

$$
-\lambda \sum_{i=1}^{3} \int_{\Omega} u_{i} \theta \frac{\partial \theta}{\partial x_{i}} \boldsymbol{d x}+\kappa \int_{\Omega}|\nabla \theta|^{2} \boldsymbol{d} \boldsymbol{x}+\alpha \int_{\partial \Omega \backslash S}|\theta|^{2} d \sigma=\lambda \int_{\Omega} \varphi(\boldsymbol{x}, \theta) \theta \boldsymbol{d} \boldsymbol{x} .
$$

Заметим, что первое слагаемое в левой части последнего равенства равно нулю. В самом деле, используя интегрирование по частям и условия

$$
\theta=0 \text { на } S, \quad \boldsymbol{u}=\mathbf{0} \text { на } \partial \Omega \backslash S, \quad \operatorname{div} \boldsymbol{u}=0 \text { в } \Omega,
$$

получаем, что

$$
\begin{aligned}
\sum_{i=1}^{3} \int_{\Omega} u_{i} \theta \frac{\partial \theta}{\partial x_{i}} \boldsymbol{d} \boldsymbol{x} & =\frac{1}{2} \sum_{i=1}^{3} \int_{\Omega} u_{i} \frac{\partial|\theta|^{2}}{\partial x_{i}} \boldsymbol{d} \boldsymbol{x}= \\
& =\frac{1}{2} \sum_{i=1}^{3} \int_{\partial \Omega} u_{i} n_{i}|\theta|^{2} d \sigma-\frac{1}{2} \sum_{i=1}^{3} \int_{\Omega} \frac{\partial u_{i}}{\partial x_{i}}|\theta|^{2} \boldsymbol{d} \boldsymbol{x}= \\
& =\frac{1}{2} \int_{\partial \Omega}(\boldsymbol{u} \cdot \mathbf{n})|\theta|^{2} d \sigma-\frac{1}{2} \int_{\Omega}(\operatorname{div} \boldsymbol{u})|\theta|^{2} \boldsymbol{d} \boldsymbol{x}=0 .
\end{aligned}
$$


Поэтому равенство (14) можно переписать следующим образом:

$$
\kappa \int_{\Omega}|\nabla \theta|^{2} \boldsymbol{d} \boldsymbol{x}=-\alpha \int_{\partial \Omega \backslash S}|\theta|^{2} d \sigma+\lambda \int_{\Omega} \varphi(\boldsymbol{x}, \theta) \theta \boldsymbol{d} \boldsymbol{x},
$$

откуда с помощью условия (C6), неравенства Коши-Буняковского и четвертого неравенства из (12) выводим:

$$
\begin{aligned}
\kappa\|\theta\|_{Y_{S}(\Omega)}^{2} & =\kappa \int_{\Omega}|\nabla \theta|^{2} \boldsymbol{d} \boldsymbol{x} \leqslant \lambda \int_{\Omega} \varphi(\boldsymbol{x}, \theta) \theta \boldsymbol{d} \boldsymbol{x} \\
\leqslant\left(\int_{\Omega}\left|\varphi_{0}\right|^{2} \boldsymbol{d} \boldsymbol{x}\right)^{1 / 2}\left(\int_{\Omega}|\theta|^{2} \boldsymbol{d} \boldsymbol{x}\right)^{1 / 2} & =\left\|\varphi_{0}\right\|_{L^{2}(\Omega)}\|\theta\|_{L^{2}(\Omega)} \leqslant \\
& \leqslant C_{4}(\Omega, S)\left\|\varphi_{0}\right\|_{L^{2}(\Omega)}\|\theta\|_{Y_{S}(\Omega)} .
\end{aligned}
$$

Следовательно,

$$
\|\theta\|_{Y_{S}(\Omega)} \leqslant \kappa^{-1} C_{4}(\Omega, S)\left\|\varphi_{0}\right\|_{L^{2}(\Omega)} .
$$

Учитывая это неравенство, из (13) нетрудно вывести следующую оценку:

$$
\begin{aligned}
\|\boldsymbol{u}\|_{\boldsymbol{V}_{S}(\Omega)} \leqslant \mu^{-1} C_{1}(\Omega, S) & \left\|p_{0}\right\|_{L^{2}(S)}+\mu^{-1} C_{2}(\Omega, S)\left\|f_{0}\right\|_{L^{2}(\Omega)}+ \\
& +\mu^{-1} \kappa^{-2} M C_{2}(\Omega, S) C_{3}^{2}(\Omega, S) C_{4}^{2}(\Omega, S)\left\|\varphi_{0}\right\|_{L^{2}(\Omega)}^{2}
\end{aligned}
$$

Дадим теперь операторную трактовку задачи (8), (9). Введем операторы:

$$
\begin{gathered}
\mathbf{A}_{1}: \boldsymbol{V}_{S}(\Omega) \rightarrow\left[\boldsymbol{V}_{S}(\Omega)\right]^{*} \\
\left\langle\mathbf{A}_{1}(\boldsymbol{w}), \boldsymbol{v}\right\rangle:=\mu \int_{\Omega} \nabla \boldsymbol{w}: \nabla \boldsymbol{v} \boldsymbol{d} \boldsymbol{x}, \\
\mathbf{A}_{2}: Y_{S}(\Omega) \rightarrow\left[Y_{S}(\Omega)\right]^{*}, \\
\left\langle\mathbf{A}_{2}(\zeta), \eta\right\rangle:=\kappa \int_{\Omega} \nabla \zeta \cdot \nabla \eta \boldsymbol{d} \boldsymbol{x}+\alpha \int_{\partial \Omega \backslash S} \zeta \eta d \sigma \\
\mathbf{A}: \boldsymbol{V}_{S}(\Omega) \times Y_{S}(\Omega) \rightarrow\left[\boldsymbol{V}_{S}(\Omega)\right]^{*} \times\left[Y_{S}(\Omega)\right]^{*} \\
\mathbf{A}(\boldsymbol{w}, \zeta):=\left(\mathbf{A}_{1}(\boldsymbol{w}), \mathbf{A}_{2}(\zeta)\right), \\
\mathbf{K}_{1}: L^{4}(\Omega) \rightarrow\left[\boldsymbol{V}_{S}(\Omega)\right]^{*} \\
\left\langle\mathbf{K}_{1}(\zeta), \boldsymbol{v}\right\rangle:=-\int_{S} p_{0}(\boldsymbol{v} \cdot \mathbf{n}) d \sigma+\int_{\Omega} \boldsymbol{f}(\boldsymbol{x}, \zeta) \cdot \boldsymbol{v} \boldsymbol{d} \boldsymbol{x} \\
\mathbf{K}_{2}: \boldsymbol{L}^{4}(\Omega) \times L^{4}(\Omega) \rightarrow\left[Y_{S}(\Omega)\right]^{*}, \\
\left\langle\mathbf{K}_{2}(\boldsymbol{w}, \zeta), \eta\right\rangle:=\sum_{i=1} \int_{\Omega} w_{i} \zeta \frac{\partial \eta}{\partial x_{i}} \boldsymbol{d x}+\int_{\Omega} \varphi(\boldsymbol{x}, \zeta) \eta \boldsymbol{d} \boldsymbol{x},
\end{gathered}
$$




$$
\begin{gathered}
\mathbf{K}: \boldsymbol{L}^{4}(\Omega) \times L^{4}(\Omega) \rightarrow\left[\boldsymbol{V}_{S}(\Omega)\right]^{*} \times\left[Y_{S}(\Omega)\right]^{*}, \\
\mathbf{K}(\boldsymbol{w}, \zeta):=\left(\mathbf{K}_{1}(\zeta), \mathbf{K}_{2}(\boldsymbol{w}, \zeta)\right), \\
\mathbf{J}: \boldsymbol{V}_{S}(\Omega) \times Y_{S}(\Omega) \rightarrow \boldsymbol{L}^{4}(\Omega) \times L^{4}(\Omega), \\
\mathbf{J}(\boldsymbol{w}, \zeta):=(\boldsymbol{w}, \zeta) .
\end{gathered}
$$

Ясно, что задача (8), (9) эквивалентна операторному уравнению

$$
\mathbf{A}(\boldsymbol{u}, \theta)=\lambda(\mathbf{K} \circ \mathbf{J})(\boldsymbol{u}, \theta) .
$$

Заметим, что

$$
\left\langle\mathbf{A}_{1}(\boldsymbol{w}), \boldsymbol{w}\right\rangle=\mu\|\boldsymbol{w}\|_{\boldsymbol{V}_{S}}^{2}, \quad\left\langle\mathbf{A}_{2}(\zeta), \zeta\right\rangle \geqslant \kappa\|\zeta\|_{Y_{S}(\Omega)}^{2}
$$

для любых $\boldsymbol{w} \in \boldsymbol{V}_{S}(\Omega)$ и $\zeta \in Y_{S}(\Omega)$. Поэтому из теоремы Лакса-Мильграма (см. [22, гл. 9, теорема 9.14]) следует, что операторы $\mathbf{A}_{1}$ и $\mathbf{A}_{2}$ непрерывно обратимы. Следовательно, непрерывно обратим и оператор $\mathbf{A}$, причем

$$
\mathbf{A}^{-1}\left(\boldsymbol{h}^{*}, \boldsymbol{g}^{*}\right)=\left(\mathbf{A}_{1}^{-1}\left(\boldsymbol{h}^{*}\right), \mathbf{A}_{2}^{-1}\left(\boldsymbol{g}^{*}\right)\right), \quad\left(\boldsymbol{h}^{*}, \boldsymbol{g}^{*}\right) \in\left[\boldsymbol{V}_{S}(\Omega)\right]^{*} \times\left[Y_{S}(\Omega)\right]^{*} .
$$

Применим $\mathbf{A}^{-1}$ к обеим частям равенства (18):

$$
(\boldsymbol{u}, \theta)=\lambda\left(\mathbf{A}^{-1} \circ \mathbf{K} \circ \mathbf{J}\right)(\boldsymbol{u}, \theta) .
$$

Оператор $\mathbf{J}$ вполне непрерывен в силу теоремы о компактности вложения соболевских пространств (см., например, [20, гл. 2, §2.6, теорема 6.1]). Кроме того, используя условия (C3)-(C6) и теорему М.А. Красносельского о непрерывности оператора суперпозиции (см. [23, гл. $1, \S 1$, предложение 1.1]), нетрудно показать, что оператор $\mathbf{K}$ непрерывен. Следовательно, оператор $\mathbf{A}^{-1} \circ \mathbf{K} \circ \mathbf{J}$ является вполне непрерывным.

Принимая во внимание полученные ранее априорные оценки (16) и (17), мы можем применить теорему Лерэ-Шаудера о неподвижной точке к операторному уравнению (19). В результате приходим к выводу о том, что уравнение

$$
(\boldsymbol{u}, \theta)=\left(\mathbf{A}^{-1} \circ \mathbf{K} \circ \mathbf{J}\right)(\boldsymbol{u}, \theta)
$$

имеет по крайней мере одно решение $\left(\boldsymbol{u}_{0}, \theta_{0}\right) \in \boldsymbol{V}_{S}(\Omega) \times Y_{S}(\Omega)$. Очевидно, что пара $\left(\boldsymbol{u}_{0}, \theta_{0}\right)$ является слабым решением задачи $(\mathrm{A})$.

Далее, подставляя $\lambda=1$ в (10) и (15), получаем энергетические равенства (6) и (7). Теорема полностью доказана.

Заключение. В работе на основе принципа Лерэ-Шаудера доказана разрешимость (в слабой постановке) смешанной краевой задачи для математической модели, описывающей стационарное ползущее течение неравномерно нагретой несжимаемой жидкости через заданную ограниченную область с локально-липшицевой границей. Кроме того, выведены энергетические равенства, которым удовлетворяют поле скоростей течения и распределение температуры в рассматриваемой области. 
Конкурирующие интересы. Заявляем, что в отношении авторства и публикации этой статьи конфликта интересов не имеем.

Авторский вклад и ответственность. Все авторы принимали участие в разработке концепции статьи и в написании рукописи. Авторы несут полную ответственность за предоставление окончательной рукописи в печать. Окончательная версия рукописи была одобрена всеми авторами.

Финансирование. Исследование выполнялось без финансирования.

Благодарность. Авторы благодарны рецензенту за тщательное прочтение статьи и ценные предложения и комментарии.

\section{Библиографический список}

1. Крейн С. Г., Чан Тху Ха. Задача протекания неравномерно нагретой вязкой жидкости // Ж. вычисл. матем. и матем. физ., 1989. Т. 29, № 8. С. 1153-1158.

2. Ковтунов Д. А. Разрешимость стационарной задачи тепловой конвекции высоковязкой жидкости // Дифференц. уравнения, 2009. Т. 45, № 1. С. 74-85.

3. Короткий А. И. Разрешимость в слабом смысле одной краевой задачи, описывающей тепловую конвекцию // Тр. ИММ УрО РАН, 2010. Т. 16, № 2. С. 121-132.

4. Алексеев Г. В. Разрешимость стационарных задач граничного управления для уравнений тепловой конвекции // Сиб. матем. журн., 1998. Т. 39, № 5. С. 982-998.

5. Фурсиков А. В., Эмануилов Ю. С. Точная управляемость уравнений Навье-Стокса и Буссинеска // УМН, 1999. Т. 54, №3(327). С. 93-146. doi : 10.4213/rm153.

6. Lee H.-C., Imanuvilov O. Yu. Analysis of optimal control problems for the 2-D stationary Boussinesq equations // J. Math. Anal. Appl., 2000. vol.242. pp. 191-211. doi: 10.1006/ jmaa.1999.6651.

7. Алексеев Г. В. Разрешимость обратных экстремальных задач для стационарных уравнений тепломассопереноса // Сиб. матем. журн., 2001. Т. 42, № 5. С. 971-991.

8. Alekseev G. V., Tereshko D. A. Stability of optimal controls for the stationary Boussinesq equations // Inter. J. Differ. Equ., 2011. vol. 2011, 535736. doi: 10.1155/2011/535736.

9. Abidi H., Zhang P. On the global well-posedness of 2-D Boussinesq system with variable viscosity // Adv. Math., 2017. vol. 305. pp. 1202-1249. doi: 10.1016/j . aim.2016.09.036.

10. Yu Y., Wu X., Tang Y. Global well-posedness for the 2D Boussinesq system with variable viscosity and damping // Math. Meth. Appl. Sci., 2018. vol. 41, no. 8. pp. 3044-3061. doi : 10. $1002 / \mathrm{mma} .4799$.

11. Li Z. Global well-posedness of the 2D Euler-Boussinesq system with stratification effects // Math. Meth. Appl. Sci., 2017. vol. 40, no.14. pp. 5212-5221. doi: 10.1002/mma. 4381.

12. Vlasova S. S., Prosviryakov E. Yu. Two-dimensional convection of an incompressible viscous fluid with the heat exchange on the free border// Vestn. Samar. Gos. Tekhn. Univ. Ser. Fiz.-Mat. Nauki [J. Samara State Tech. Univ., Ser. Phys. Math. Sci.], 2016. vol. 20, no. 3. pp. 567-577. doi: 10.14498/vsgtu1483.

13. Privalova V. V., Prosviryakov E. Yu. Couette-Hiemenz exact solutions for the steady creeping convective flow of a viscous incompressible fluid, with allowance made for heat recovery // Vestn. Samar. Gos. Tekhn. Univ. Ser. Fiz.-Mat. Nauki [J. Samara State Tech. Univ., Ser. Phys. Math. Sci.], 2018. vol. 22, no. 3. pp. 532-548. doi: 10.14498/vsgtu1638.

14. Бурмашева Н. В., Просвиряков Е. Ю. Крупномасштабная слоистая стационарная конвекция вязкой несжимаемой жидкости под действием касательных напряжений на верхней границе. Исследование поля скоростей // Вестн. Сам. гос. техн. ун-та. Сер. Физ.-мат. науки, 2017. Т. 21, №1. С. 180-196. doi: 10.14498/vsgtu1527.

15. Бурмашева Н. В., Просвиряков Е. Ю. Крупномасштабная слоистая стационарная конвекция вязкой несжимаемой жидкости под действием касательных напряжений на верхней границе. Исследование полей температуры и давления // Becmн. Сам. гос. техн. ун-та. Сер. Физ.-мат. науки, 2017. Т. 21, №4. С. 736-751. doi: 10.14498/ vsgtu1568. 
16. Рагулин В. В. К задаче о протекании вязкой жидкости сквозь ограниченную область при заданном перепаде давления и напора // Динамика сплошной среды, 1976. Т. 27. C. $78-92$.

17. Conca C., Murat F., Pironneau O. The Stokes and Navier-Stokes equations with boundary conditions involving the pressure// Japan. J. Math., 1994. vol. 20. pp. 279-318. doi: 10 . 4099/math1924.20.279.

18. Marušić S. On the Navier-Stokes system with pressure boundary condition// Ann. Univ. Ferrara, 2007. vol. 53. pp. 319-331. doi: 10.1007/s11565-007-0024-y.

19. Bertoluzza S., Chabannes V., Prud'homme C., Szopos M. Boundary conditions involving pressure for the Stokes problem and applications in computational hemodynamics // Comput. Methods Appl. Mech. Eng., 2017. vol.322. pp. 58-80. doi:10.1016/j.cma.2017.04. 024.

20. Nečas J. Direct Methods in the Theory of Elliptic Equations. Heidelberg: Springer, 2012. xvi+372 pp. doi : 10.1007/978-3-642-10455-8.

21. Ладыженская О. А. Математические вопросы динамики вязкой несжимаемой жидкости. М.: Наука, 1970. 288 с.

22. Renardy M., Rogers R. An Introduction to Partial Differential Equations, 2nd edition. New York: Springer-Verlag, 2004. xiv+434 pp. doi : 10.1007/b97427.

23. Скрыпник И. В. Методы исследования нелинейных эллиптических граничных задач. М.: Наука, 1990. 488 c. 
MSC: 35Q35, 35Q79, 35A01

\title{
On a mathematical model of non-isothermal creeping flows of a fluid through a given domain
}

\author{
A. A. Domnich ${ }^{1}$, E. S. Baranovskii ${ }^{2}$, M. A. Artemov ${ }^{2}$ \\ 1 Russian Air Force Military Educational and Scientific Center of the \\ "N. E. Zhukovskiy and Yu. A. Gagarin Air Force Academy", \\ 54 a, Staryh Bolshevikov, Voronezh, 394064, Russian Federation. \\ 2 Voronezh State University, \\ 1, Universitetskaya pl., Voronezh, 394018, Russian Federation.
}

\begin{abstract}
We study a mathematical model describing steady creeping flows of a non-uniformly heated incompressible fluid through a bounded 3D domain with locally Lipschitz boundary. The model under consideration is a system of second-order nonlinear partial differential equations with mixed boundary conditions. On in-flow and out-flow parts of the boundary the pressure, the temperature and the tangential component of the velocity field are prescribed, while on impermeable solid walls the no-slip condition and a Robin-type condition for the temperature are used. For this boundary-value problem, we introduce the concept of a weak solution (a pair "velocitytemperature"), which is defined as a solution to some system of integral equations. The main result of the work is a theorem on the existence of weak solutions in a subspace of the Cartesian product of two Sobolev's spaces. To prove this theorem, we give an operator interpretation of the boundary value problem, derive a priori estimates of solutions, and apply the LeraySchauder fixed point theorem. Moreover, energy equalities are established for weak solutions.
\end{abstract}

Keywords: flux problem, non-isothermal flows, creeping flows, mixed boundary conditions, weak solutions.

Received: $15^{\text {th }}$ June, $2019 /$ Revised: $14^{\text {th }}$ July, $2019 /$

Accepted: $16^{\text {th }}$ September, $2019 /$ First online: $14^{\text {th }}$ October, 2019

\section{Research Article}

() (1) The content is published under the terms of the Creative Commons Attribution 4.0 International License (http://creativecommons.org/licenses/by/4.0/)

Please cite this article in press as:

Domnich A. A., Baranovskii E. S., Artemov M. A. On a mathematical model of nonisothermal creeping flows of a fluid through a given domain, Vestn. Samar. Gos. Tekhn. Univ., Ser. Fiz.-Mat. Nauki [J. Samara State Tech. Univ., Ser. Phys. Math. Sci.], 2019, vol. 23, no. 3, pp. 417-419. doi: 10.14498/vsgtu1713 (In Russian).

\section{Authors' Details:}

Anastasia A. Domnich (1) https://orcid.org/0000-0001-5231-4907

Lecturer; Dept. of Higher Mathematics; e-mail: andomnich@inbox.ru

Evgenii S. Baranovskii (D) https://orcid.org/0000-0002-1514-4475

Cand. Phys. \& Math. Sci.; Associate Professor; Dept. of Applied Mathematics, Informatics, and Mechanics; e-mail: esbaranovskii@gmail.com

Mikhail A. Artemov (10 https://orcid.org/0000-0001-8356-5418

Dr. Phys. \& Math. Sci., Professor; Head of Chair; Dept. of Applied Mathematics, Informatics, and Mechanics; e-mail: artemov_m_a@mail.ru 
Competing interests. We declare that we have no conflicts of interests with the authorship and publication of this article.

Authors' contributions and responsibilities. Each author has participated in the article concept development and in the manuscript writing. The authors are absolutely responsible for submitting the final manuscript in print. Each author has approved the final version of manuscript.

Funding. This research received no specific grant from any funding agency in the public, commercial, or not-for-profit sectors.

\section{References}

1. Krein S. G., Chan Tkhu Kha. The problem of the flow of a non-uniformly heated viscous fluid, U.S.S.R. Comput. Math. Math. Phys., 1989, vol. 29, no. 4, pp. 127-131. doi: 10.1016/ 0041-5553(89) 90127-4.

2. Kovtunov D. A. Solvability of the stationary heat convection problem for a high-viscosity fluid, Differ. Equ., 2009, vol.45, no. 1, pp. 73-85. doi: 10.1134/S0012266109010091.

3. Korotkii A. I. Solution in weak sense of a boundary value problem describing thermal convection, Trudy Inst. Mat. i Mekh. UrO RAN, 2010, vol. 16, no. 2, pp. 121-132 (In Russian).

4. Alekseev G. V. Solvability of stationary problems of boundary control for thermal convection equations, Siberian Math. J., 1998, vol. 39, no. 5, pp. 844-858. doi : 10.1007/BF02672906.

5. Fursikov A. V., Emanuilov Yu. S. Exact controllability of the Navier-Stokes and Boussinesq equations, Russian Math. Surveys, 1999, vol.54, no. 3, pp. 565-618. doi: 10.1070/ rm1999v054n03ABEH000153.

6. Lee H.-C., Imanuvilov O. Yu. Analysis of optimal control problems for the 2-D stationary Boussinesq equations, J. Math. Anal. Appl., 2000, vol.242, pp. 191-211. doi:10.1006/ jmaa.1999.6651.

7. Alekseev G. V. Solvability of inverse extremal problems for stationary heat and mass transfer equations, Siberian Math. J., 2001, vol.42, no.5, pp. 811-827. doi: 10.1023/A: 1011940606843.

8. Alekseev G. V., Tereshko D. A. Stability of optimal controls for the stationary Boussinesq equations, Inter. J. Differ. Equ., 2011, vol. 2011, 535736. doi: 10.1155/2011/535736.

9. Abidi H., Zhang P. On the global well-posedness of 2-D Boussinesq system with variable viscosity, Adv. Math., 2017, vol.305, pp. 1202-1249. doi: 10.1016/j.aim.2016.09.036.

10. Yu Y., Wu X., Tang Y. Global well-posedness for the 2D Boussinesq system with variable viscosity and damping, Math. Meth. Appl. Sci., 2018, vol. 41, no. 8, pp. 3044-3061. doi: 10. $1002 / \mathrm{mma} .4799$.

11. Li Z. Global well-posedness of the 2D Euler-Boussinesq system with stratification effects, Math. Meth. Appl. Sci., 2017, vol. 40, no. 14, pp. 5212-5221. doi: 10.1002/mma. 4381.

12. Vlasova S. S., Prosviryakov E. Yu. Two-dimensional convection of an incompressible viscous fluid with the heat exchange on the free border, Vestn. Samar. Gos. Tekhn. Univ. Ser. Fiz.-Mat. Nauki [J. Samara State Tech. Univ., Ser. Phys. Math. Sci.], 2016, vol. 20, no. 3, pp. 567-577. doi: 10.14498/vsgtu1483.

13. Privalova V. V., Prosviryakov E. Yu. Couette-Hiemenz exact solutions for the steady creeping convective flow of a viscous incompressible fluid, with allowance made for heat recovery, Vestn. Samar. Gos. Tekhn. Univ. Ser. Fiz.-Mat. Nauki [J. Samara State Tech. Univ., Ser. Phys. Math. Sci.], 2018, vol. 22, no. 3, pp. 532-548. doi: 10.14498/vsgtu1638.

14. Burmasheva N. V., Prosviryakov E. Yu. A large-scale layered stationary convection of a incompressible viscous fluid under the action of shear stresses at the upper boundary. Velocity field investigation, Vestn. Samar. Gos. Tekhn. Univ., Ser. Fiz.-Mat. Nauki [J. Samara State Tech. Univ., Ser. Phys. Math. Sci.], 2017, vol.21, no.1, pp. 180-196 (In Russian). doi : $10.14498 /$ vsgtu1527. 
15. Burmasheva N. V., Prosviryakov E. Yu. A large-scale layered stationary convection of a incompressible viscous fluid under the action of shear stresses at the upper boundary. Temperature and presure field investigation, Vestn. Samar. Gos. Tekhn. Univ., Ser. Fiz.-Mat. Nauki [J. Samara State Tech. Univ., Ser. Phys. Math. Sci.], 2017, vol. 21, no. 4, pp. 736-751 (In Russian). doi: 10.14498/vsgtu1568.

16. Ragulin V. V. To the problem of viscous fluid flow through bounded region at given pressure of head drop, Dinamika sploshnoy sredy, 1976, vol. 27, pp. 78-92 (In Russian).

17. Conca C., Murat F., Pironneau O. The Stokes and Navier-Stokes equations with boundary conditions involving the pressure, Japan. J. Math., 1994, vol.20, pp. 279-318. doi: 10.4099/ math1924.20.279.

18. Marušić S. On the Navier-Stokes system with pressure boundary condition, Ann. Univ. Ferrara, 2007, vol. 53, pp. 319-331. doi: 10.1007/s11565-007-0024-y.

19. Bertoluzza S., Chabannes V., Prud'homme C., Szopos M. Boundary conditions involving pressure for the Stokes problem and applications in computational hemodynamics, Comput. Methods Appl. Mech. Eng., 2017, vol.322, pp. 58-80. doi: 10.1016/j.cma.2017.04.024.

20. Nečas J. Direct Methods in the Theory of Elliptic Equations. Heidelberg, Springer, 2012, $\mathrm{xvi}+372$ pp. doi : 10.1007/978-3-642-10455-8.

21. Ladyzhenskaya O. A. The Mathematical Theory of Viscous Incompressible Flow. New York, London, Paris, Gordon and Breach, 1969, xviii +224 pp.

22. Renardy M., Rogers R. An Introduction to Partial Differential Equations, 2nd edition. New York, Springer-Verlag, 2004, xiv+434 pp. doi : 10.1007/b97427.

23. Skrypnik I. V. Methods for Analysis of Nonlinear Elliptic Boundary Value Problems. Providence, RI, Amer. Math. Soc., 1994, xii+348 pp. 